\title{
Behind the Scenes Of Cns Vasculitis: A Rare Case of Cns Vasculitis with Two Primary Malignancies
}

\author{
Savvina Prapiadou ${ }^{1}$, Daniel Woo $^{2}$, Adam Koss ${ }^{2}$, Andrew Creed ${ }^{2}$, Rina Mina ${ }^{3}$, Priyanka Vashisht ${ }^{3}$, Kristina \\ Brannock $^{4}$, Mosaab Mohameden ${ }^{3 *}$
}

${ }^{1}$ Department of Medicine, University of Patras College of Medicine, Greece

${ }^{2}$ Department of Neurology and Rehabilitation Medicine, University of Cincinnati College of Medicine, USA

${ }^{3}$ Department of Internal Medicine, Rheumatology Division, University of Cincinnati College of Medicine, USA

${ }^{4}$ Department of Pathology, University of Cincinnati College of Medicine, USA

*Corresponding author: Mosaab Mohameden, Department of Internal Medicine, Rheumatology Division, University of Cincinnati College of Medicine, USA.

Received Date: July 09, 2021

Published Date: October 05, 2021

Abstract

Purpose of Review

To highlight the importance of a broad differential diagnosis, including malignancy, in patients with central nervous system vasculitis (CNSV) presenting as multiple, recurrent cryptogenic strokes.

Recent Findings

We describe a rare entity, CNSV in the setting of two underlying neoplastic disorders and a chorioretinal inflammatory disease. The clinical presentation, imaging study results, histopathologic features, and response to treatment are reported.

Summary

Cancer-associated CNSV is a rare cause of recurrent cryptogenic strokes. This case highlights the importance of clinical suspicion of and comprehensive work-up for neoplasms in a patient presenting with brain lesions consistent with CNSV.

Keywords: Vasculitis; Infarction; Visual loss; Retina; Paraneoplastic syndrome

Abbreviations

APMPPE- Acute posterior multifocal placoid pigment epitheliopathy

CNSV- Central Nervous system Vasculitis

MRI- magnetic resonance imaging

MRA- magnetic resonance angiography

ANA- anti-nuclear antibody

CSF- Cerebrospinal fluid

PET- positron emission tomography

CT - computerized tomography

CYC- cyclophosphamide 


\section{Introduction}

Acute posterior multifocal placoid pigment epitheliopathy (APMPPE) is a rare, self-limiting chorioretinal inflammatory disorder that is often characterized by a flu-like prodrome and usually presents in young adults [1]. Neurological complications are rare but potentially fatal manifestations of APMPPE that have been previously reported in 56 cases, with the most common being CNSV (50\% of the cases) [2]. CNSV is a diagnostically challenging disease and has been rarely associated with malignancies, especially with Hodgkin lymphoma [3].

We report one patient with APMPPE and neurological symptoms consistent with CNSV, who was diagnosed with two primary malignancies during the medical work-up for CNSV.

\section{Case Presentation}

A 59-year-old female presented to the hospital with bilateral blurry vision, floaters and light flashes that persisted over a twoweek period. These symptoms were preceded by holocephalic aching headaches associated with temporal tenderness two weeks prior to the vision symptoms. The patient has a medical history of recurrent herpes zoster, hyperlipidemia, and type 2 diabetes mellitus. Review of systems was notable for fatigue, fever, chills, night sweats, dry mouth, and weight loss of 64 pounds in the past year. Physical exam revealed normal vital signs, a palpable left submandibular lymph node, impaired visual acuity to counting fingers at 3 feet in the left eye and 20/200 in the right eye, and fundus exam revealed yellow-white placoid lesions with areas of chorioretinal atrophy (Figures 1-4) The remainder of the eye exam was unremarkable. Neurological examination revealed impaired range of central visual field and decreased sensation to pinprick in the right cranial nerve V3 (mandibular) distribution and right upper extremity. Brain magnetic resonance imaging (MRI) showed a subacute lacunar infarct in the dorsal left putamen/capsular junction and minimal white matter chronic microvascular ischemic disease (Figure 5). Cranial magnetic resonance angiography (MRA) demonstrated no evidence of flow-limiting stenosis or occlusion. The patient was treated with atorvastatin $40 \mathrm{mg}$ and aspirin 325 mg for secondary stroke prevention.

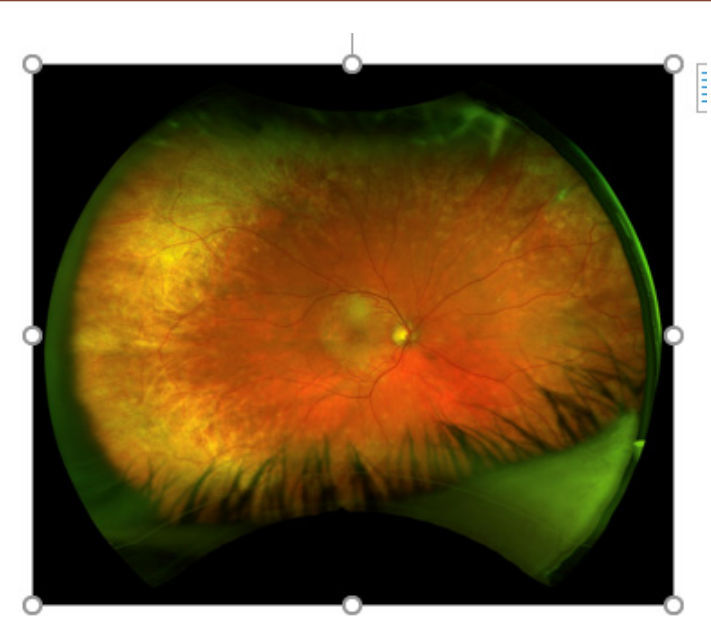

Figure 1: Right Eye Fundoscopy: showing yellow white placoid lesions with evidence of chorioretinal atrophy consistent with APMPPE.

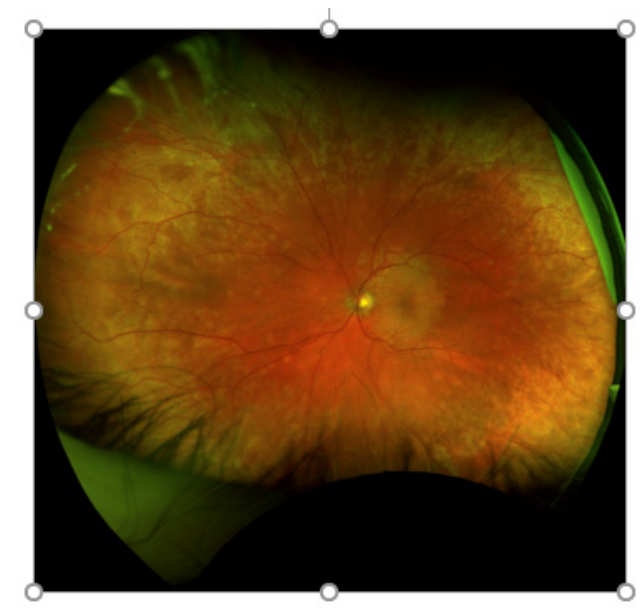

Figure 2: Left eye Fundoscopy: showing yellow white placoid lesions with evidence of chorioretinal atrophy consistent with APMPPE. 


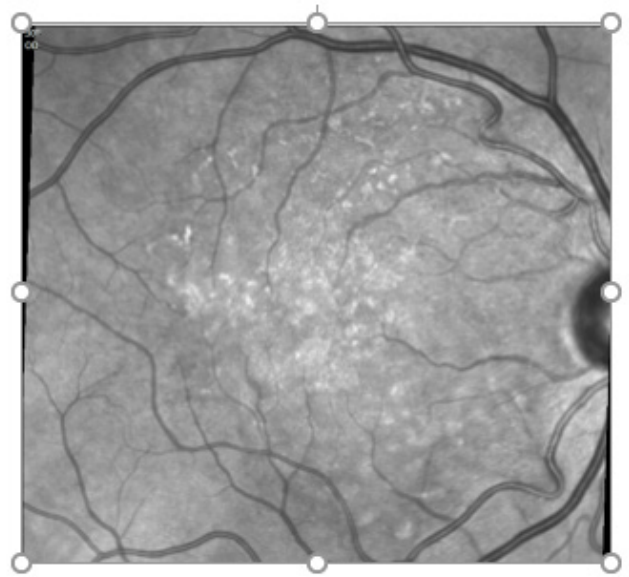

Figure 3: Right eye Fluorescein angiography: showing patchy areas of hyperfluorescence consistent with APMPPE.

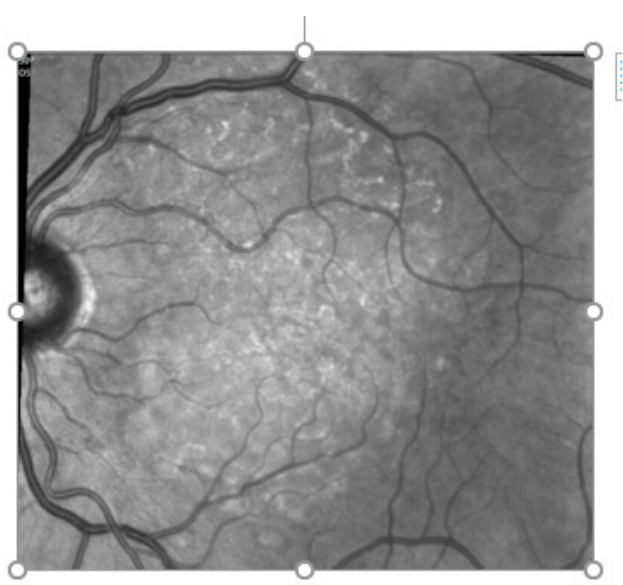

Figure 4: Left eye Fluorescein angiography: showing patchy areas of hyper fluorescence consistent with APMPPE.

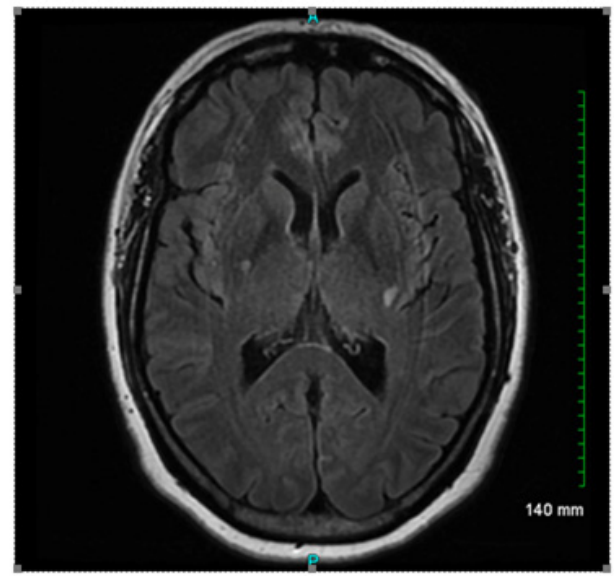

Figure 5: MRI of the brain revealed a subacute lacunar infarct in the dorsal left putamen/capsular junction.

Ophthalmologic findings triggered a concern for cerebral vasculitis, thus immunologic workup was done which was unremarkable except for a weakly positive anti-nuclear antibody
(ANA) (titer of 1:160). The results of the tests for anti-dsDNA and anti-extractable nuclear antigen antibodies, viral hepatitis B and C, and HIV screening were all negative. 
Laboratory studies at presentation demonstrated mildly elevated inflammatory markers (Table 1). Cerebrospinal fluid (CSF) demonstrated lymphocytic pleocytosis and elevated protein levels (Table 2). CSF studies for varicella zoster virus PCR, IgG, and IgM were negative. Transthoracic echocardiogram and

Table 1: Relevant lab studies.

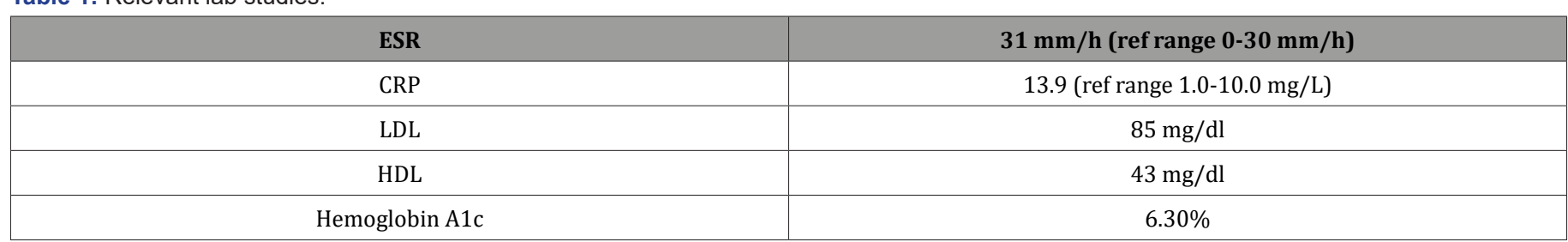

Table 2: CSF Findings.

\begin{tabular}{|c|c|c|}
\hline CSF (Ref Range) & On presentation & Colorless \\
\hline Appearance & Colorless & Clear \\
\hline Clarity & Clear & 0 \\
\hline White cells (per mm ${ }^{3}$ ) (0-5) & 21 & - \\
\hline Lymphocytes (\%) (40-80\%) & 76 & 69 \\
\hline Red Cells (per mm ${ }^{3}$ ) (0-10) & 19 & 47 \\
\hline Protein (mg/dl) $15-45$ & 54 & 98 \\
\hline Glucose (mg/dL) $40-70$ & 64 & 7 \\
\hline CSF/serum index (0-8) & 6 & None seen \\
\hline Oligoclonal IgG bands & None seen & 0.6 \\
\hline IgG index (0.0-0.7) & 0.6 & 0.1 \\
\hline IgG synthesis (-9.9-3.3 $\mathrm{mg} /$ day) & 0.04 & \\
\hline
\end{tabular}

Catheter-based angiogram of the brain was negative for vasculitic changes. Given the lack of definite diagnosis, a brain biopsy was ordered. However, a pre-operative head CT scan showed multiple interval lacunar infarcts within the right periventricular corona radiata, right cerebellum, mesial left temporal lobe, and left occipital lobe, and an evolving subacute infarct within the left basal ganglia. Additionally, whole body PET CT imaging showed a region with increased uptake in the right breast with associated hypermetabolic right axillary lymphadenopathy and left submandibular lymph node, but no evidence of large-vessel vasculitis (Figure 6). At that time the brain biopsy was aborted pending work up for these lesions, prednisone was titrated to $100 \mathrm{mg}$ by mouth daily, and the patient was discharged to facilitate necessary outpatient oncologic workup, including breast biopsy.

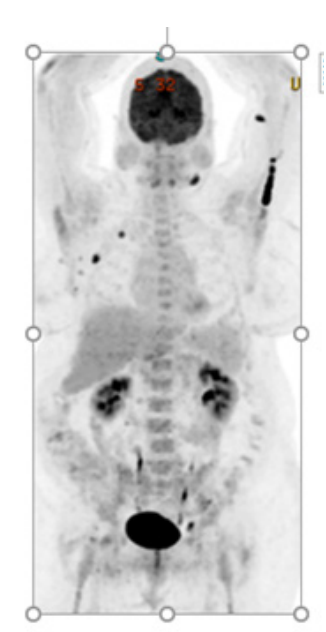

Figure 6: Whole body PET scan showed increased uptake in the right breast, right axillary lymph nodes, and left submandibular lymph node with no evidence of vasculitis. 


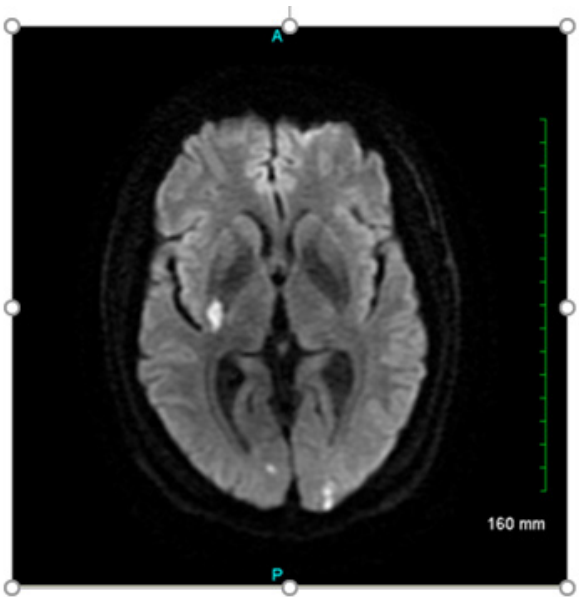

Figure 7: Interval brain MRI 4 days after presentation revealed new acute infarcts in the superior frontal gyrus and bilaterally in the occipital lobe.

Four days later, immediately following her breast biopsy, the patient presented again with an episode of numbness and paresthesia on the left side of her body, speech difficulty, and facial droop. A repeat MRI confirmed the presence of multiple new acute infarcts in the superior frontal gyrus and bilaterally in the occipital lobe (Figure 7).

The breast biopsy revealed invasive ductal carcinoma. A needle core biopsy of the patient's submandibular lymph node was consistent with follicular lymphoma; however the sample was too scant for grading. An open brain biopsy (right occipital craniotomy and biopsy of dura and brain with neuro navigation) was performed, which showed no evidence of neoplastic process or definite features of vasculitis. Repeat catheter angiography of the brain demonstrated multifocal regions of vessel wall narrowing leading to occlusion, most pronounced in distal bilateral middle cerebral arteries and distal left posterior cerebral artery territories concerning for vasculitis or other cause of vasospasm. No verapamil was administered due to recent stroke and risk of reperfusion hemorrhage.

Based on discussion with oncology, both breast malignancy and follicular lymphoma were thought to be indolent and thus a decision was made to start induction immunosuppression for CNSV.

The patient was started on cyclophosphamide (CYC) $600 \mathrm{mg} /$ m2 IV every 4 weeks along with tapering the prednisone, which was well-tolerated for 3 doses of CYC. However, one week after her third dose she had an episode of right leg weakness and numbness and presented to the emergency room, where brain MRI showed new subacute/acute infarcts in both cerebral hemispheres and the left genu of the corpus callosum. CT angiogram showed new multifocal areas of moderate to severe narrowing of the bilateral pericallosal arteries and posterior cerebral arteries. Transthoracic echocardiogram was again negative for acute or chronic abnormalities and repeat CSF studies showed normal leukocyte count and protein level.

At this point, the decision was made to add rituximab $375 \mathrm{mg} /$ $\mathrm{m} 2$ IV weekly to her CYC therapy and increase the prednisone dose to $60 \mathrm{mg}$. At the time of submission of this case report, the patient had received a total of 5 doses of CYC and 6 doses of rituximab. Clinically, the patient did well with no recurrence of neurologic deficits.

\section{Discussion}

CNSV was found in some studies to be associated with APMPPE. The mechanism that leads to this association is unknown but is often attributed to an underlying vasculitis process [2]. Our experience here demonstrates the difficulty of diagnosing CNSV, a rare disease that is sometimes associated with other pathologies. Ambiguous clinical presentation and radiographic imaging can often mimic other disease processes and can present diagnostic and therapeutic challenges. Angiography has a sensitivity for diagnosing CNSV between $50 \%$ and $90 \%$ while some report the sensitivity of brain biopsy to be less than $50 \%$, although the latter might be helpful in excluding the presence of other syndromes [4]. In our case, the diagnostic interventions were further complicated by the discovery of two primary malignancies as associated and potentially causative pathologies.

To our knowledge there is only one other report in the literature of a case of APMPPE associated with a malignancy. However, in that case the patient presented differently. He was diagnosed with and treated for metastatic clear cell renal carcinoma before the APMPPE was diagnosed. The development of APMPPE was proposed to be a result of the spreading of tumor-originated circulating immune complexes [5]. CNSV has been rarely associated with malignancy. CNSV has been previously reported in a patient who presented with intractable seizures and was later diagnosed with invasive lobular carcinoma of the breast [6]. Two additional patients presenting with CNSV after receiving treatment for malignant vaginal squamous 
cell carcinoma and invasive breast ductal carcinoma responded to methylprednisolone/plasmapheresis and to prednisone, respectively [7].

A study looking at patients diagnosed with any type of vasculitis and lymphoma over a 32-year period found the incidence of lymphoma presenting together with CNSV to be $10 / 168$, or $5.9 \%$. All of the patients in that study were treated with prednisone and two patients also received cyclophosphamide. Overall, 6/10 patients responded to therapy and showed almost no residual deficits [3]. Significant in our case is the simultaneous presence of two primary malignancies in addition to APMPPE and a CNSV process. Cyclophosphamide may be an appropriate firstline treatment in a patient with CNSV. However, in the setting of underlying malignancy, our patient did not respond well to cyclophosphamide despite adequate dosing for 12 weeks. The addition of rituximab to cyclophosphamide was possibly beneficial due to the underlying follicular lymphoma, pointing to the possibility of a paraneoplastic etiology of the CNSV. The relationship between systemic malignancies and CNSV continues to be difficult to assess. A cell-mediated immune process triggered by abnormal serum proteins or tumor antigens has been suggested as a possible etiopathogenetic mechanism [6,7]. Treatment recommendations and patient outcomes vary in the literature. Additionally, these clinical entities are often difficult to diagnose which leads to further problems in determining the optimal treatment plan. Our case demonstrates the importance of considering a broad differential in a patient presenting with APMPPE and atypical persistent neurologic symptoms. Further studies are needed to help guide treatment for CNSV when associated with malignancy Appendix 1.

\section{Conflict of Interest}

The authors report no financial disclosures

\section{Acknowledgement}

None.

\section{References}

1. Jones NP (1995) Acute posterior multifocal placoid pigment epitheliopathy. Br J Ophthalmol 79(4): 384-389.

2. Algahtani H, Alkhotani A, Shirah B (2016) Neurological Manifestations of Acute Posterior Multifocal Placoid Pigment Epitheliopathy. J Clin Neurol 12(4): 460-467.

3. Salvarani C, Brown RD, Christianson TJH (2018) Primary central nervous system vasculitis associated with lymphoma. Neurology 90(10): e847.

4. Birnbaum J, Hellmann DB (2009) Primary Angiitis of the Central Nervous System. Arch Neurol 66(6): 704-709.

5. Parmeggiani F, Costagliola C, D Angelo S, Incorvaia C, Perri P, et al. (2004) Clear Cell Renal Cell Carcinoma Associated with Bilateral Atypical Acute Posterior Multifocal Placoid Pigment Epitheliopathy. Oncology 66(6): 502-509.

6. Taccone FS, Salmon I, Marechal R, Blecic SA (2007) Paraneoplastic vasculitis of central nervous system presenting as recurrent cryptogenic stroke. Int J Clin Oncol 12(2): 155-159.

7. Sheehan J, Tate J, Mott R, Geer C, Wolfe, et al. (2018). Pearls \& Oy-sters: The critical role of histopathology in diagnosing cancer-associated necrotizing CNS vasculitis. Neurology 90(17): 808-811. 